\title{
La nomenclatura botánica en el jardín verbal de María Victoria Atencia ${ }^{1}$
}

\author{
Antonio Portela Lopa \\ Universidad de Burgos
}

Título: La nomenclatura botánica en el jardín verbal de María Victoria Atencia.

Resumen: El léxico botánico ocupa un lugar privilegiado dentro de la obra de María Victoria Atencia. Destaca un nutrido corpus de poemas dedicados a jardines, muchos de su Málaga natal, que conviven con un representativo grupo de vergeles foráneos. Junto a estos espacios, públicos y privados, crece una gran variedad de plantas significadas por medio de distintos planos léxicos (desde el poético hasta el científico, pasando por el popular). Este artículo recorre el jardín verbal de Atencia, deteniéndose en el sugestivo uso de la nomenclatura botánica en cinco poemas: "Palo borracho", "Dracúnculo", "Rosa de Jericó”, "Le Temps" y "Datura suaveolens".

Palabras clave: María Victoria Atencia, léxico, Botánica, jardín.

Fecha de recepción: 20/4/2021.

Fecha de aceptación: 13/5/2021.
Title: Botanical Nomenclature in the Verbal Garden of Maria Victoria Atencia.

Abstract: Botanical lexicon occupies a prominent place in María Victoria Atencia’s poetry. Her work includes a large corpus of poems dedicated to gardens, many of them in her native Málaga, which coexist with a representative group of foreign orchards. Alongside these public and private spaces, a wide variety of plants are represented, named from different simultaneous planes of language (from the poetic to the scientific one, including popular language). This paper will take a look at Atencia's verbal garden throughout his work, paying special attention to the interesting use of botanical nomenclature present in five poems: "Palo borracho", "Dracúnculo", "Rosa de Jericó", "Le Temps" and "Datura suaveolens".

Key words: María Victoria Atencia, Lexicon, Botany, Garden.

Date of Receipt: 20/4/2021.

Date of Approval: 13/5/2021.

1 Este trabajo forma parte del Proyecto de Investigación HELEA, "Hibridismo y Elogio en la España Áurea", PGC2018-095206-B-I00, dirigido por Jesús Ponce Cárdenas (UCM), financiando por el Ministerio de Ciencia, Innovación y Universidades y el Fondo Europeo de Desarrollo Regional. 


\section{INTRODUCCIÓN}

Cuando el lenguaje coincide con el jardín delimita un corpus, desplegando un mapa en relieve de sentidos y referencias. En dichos casos, puede considerarse que en el lenguaje se suceden los nombres del reino vegetal de una manera similar al itinerario por el jardín. Cuando se habla de un jardín, se construye una zona del discurso equivalente a ese espacio delimitado. Tal igualdad entre el lenguaje y seres que designa está hecha de equivalencias perfectas entre la palabra y la cosa, entre cada uno de los nombres y cada una de las plantas que componen lo más excepcional de un jardín propio, ya sea público o privado.

Más misteriosos aún resultan estos lazos en el campo del lenguaje poético. Así sucede con la nomenclatura botánica en la obra de María Victoria Atencia, que vamos a denominar su jardín verbal, porque constituye un corpus de sustantivos vegetales con plenitud de símbolo, y de adjetivos que redefinen y acotan sus planos referenciales. En estas páginas los estudiaremos desde la ladera lexicológica, otorgando a cada uno de ellos la máxima condición significativa. Tal y como tendremos oportunidad de comprobar, cuando en la obra de Atencia se habla de jardines, los nombres de las plantas (en diversos planos del lenguaje) serán los protagonistas. Su naturaleza (espirituales, terrenales, verbales), pues, se publica de un modo esencialmente sustantivo, designando prístinamente los seres que en ellos crecen.

Una característica esencial de la terminología botánica en Atencia se cifra en el empleo singular y simultáneo de distintas variedades del lenguaje: desde el popular hasta el científico. Siente un gran interés por la taxonomía y acudirá a su nomenclatura oficial en los lugares que sondeamos en estas páginas, a veces en compañía de sus equivalentes comunes. He aquí todo un subcorpus que conforma el eje de nuestro trabajo. En este sentido, seguimos el método de Callebat en su clásico estudio sobre la rosa ${ }^{2}$, que atendió desde la nomenclatura científica y el origen latino del término hasta el desarrollo simbólico que alcanza en los clásicos. En nuestro caso, haremos un recorrido por el vocabulario científico, popular y personalísimo de María Victoria Atencia, dueńa de todas las posibilidades de la lengua cuando se trata de referir a los seres con cuya belleza se construyen los jardines.

2 Louis Callebat, "Rosa, la rose", Voces, 3 (1992), pp. 21-29. 
Junto al citado estudio de Callebat, también nos fundaremos en el monográfico de la revista Creneida (2013) sobre las flores en la literatura española (aunque sin limitarnos a ellas), toda vez que abrió el camino a posteriores análisis ${ }^{3}$. De su selección de artículos reseñamos el de Rosso, cuyo paseo por el jardín de Altolaguirre puede considerarse otro modelo para estas páginas ${ }^{4}$. Tras estas aportaciones, el estudio de la presencia de elementos botánicos en la poesía comienza a constituirse ya como género filológico de cierto alcance, situado a medio camino (o en la mitad de un sendero de jardín) entre la lexicología y la botánica. Varias tesis defendidas en los últimos años informan del interés académico por la presencia de lo vegetal en las letras, dando cabida entre ellas a la lectura ecológica de la literatura: Peña Álvarez se centra en la aparición de las flores en el Siglo de Oro español'5; Assaad Cherif abundó sobre el mismo asunto en la obra de Alberti ${ }^{6}$; y la más reciente de todas, la de Yánez Velasco ${ }^{7}$, abre una perspectiva internacional más teórica para delimitar el simbolismo del "bosque literario", como otro de los espacios poblados por lo vegetal (objeto asimismo del interés de Atencia, aunque en menor medida que el jardín).

Esta perspectiva gana estatura si nos ceñimos al círculo más próximo de nuestra poeta. Un estudio publicado sobre Pablo García Baena, en el haber de Sánchez Zamarreño ${ }^{8}$, sondea el opulento y muy preciso léxico vegetal en los versos del cordobés. Recordemos que Atienza mantuvo una larga amistad con García Baena y que compartieron tanto su gusto por las plantas como la exactitud a la hora de cantarlas.

La familiaridad con el léxico botánico que evidencia la obra de Atencia obedece a una íntima curiosidad por la belleza, abundancia y riqueza del

3 Creneida: Anuario de Literaturas Hispánicas: "Las flores en la literatura española", 1 (2013).

4 María Rosso Gallo, "En El Jardín de Manuel Altolaguirre", Creneida: Anuario de Literaturas Hispánicas, 1 (2013), pp. 160-78.

5 Javier de la Peña Álvarez, Flores en la poesía española del Renacimiento y Barroco, Madrid, Universidad Complutense, 2009 [tesis doctoral].

6 Omar Nirvana Assaad Cherif, El mundo vegetal en la poesía de Rafael Alberti, Madrid, Universidad Complutense, 2003 [tesis doctoral].

7 Marcos Yáñez Velasco, El bosque literario. Genealogía de un paisaje simbólico, Barcelona, Universitat Pompeu Fabra, 2018 [tesis doctoral].

8 Antonio Sánchez Zamarreńo, "El Barroquismo de la flora en la poesía de Pablo García Baena”, en Homenaje a José María Martínez Cachero: Investigación y Crítica, Oviedo, Universidad de Oviedo, 2000, III, pp. 507-520. 
reino vegetal. Pero pueden detallarse otras noticias bio-bibliográficas que nos arrojan luz sobre su interés lingüístico' ${ }^{9}$ El temprano encuentro con la obra de Dioscórides es sin duda el más llamativo, hasta el punto de explicar la incorporación del lenguaje científico al poético. Sin ir más lejos, Rafael León, marido de la escritora, homenajeó la obra de Dioscórides en un poemario ${ }^{10}$. Algunos de los textos que lo integran comparten con la obra del griego su apego a la descripción sensorial y empírica a un tiempo de los vegetales; un enfoque que también aflora en Atencia. Pero la literatura botánica forma parte de sus lecturas, como anuncia en el temprano "Villa Jaraba"11. Tras la descripción de aquella villa "colgada / en el sueño", la poeta dirige la mirada hacia los ranúnculos, y apunta: "solo / los he visto en los libros de botánica” (vv. 5-6).

En la poética de Atencia, al menos en la que dedica a los verdores, se dan la mano estos dos planos del saber, cuyos lenguajes fueron uno en algún momento de la cultura occidental. Tendremos, pues, el latín y el griego acompańando a la lengua popular. Quizá dicha voluntad conciliadora también pueda considerarse un rasgo generacional, a juzgar por la importancia que cobra el léxico científico en algunos de sus coetáneos. El gallego Ernesto Guerra da Cal, con quien Atencia mantuvo correspondencia (y a quien dedica el poema "Jardín de Portugal"), trató ya de acercar el léxico científico al poético" ${ }^{12}$. Y Antonio Gamoneda evidenció en el Libro de los venenos (1995) su cercanía con el Dioscórides traducido por Andrés Laguna ${ }^{13}$.

9 He tratado algunos de estos aspectos en un estudio anterior: Antonio Portela Lopa, "Una lectura del Siglo de Oro: el perfil de la azucena", en La poesía de María Victoria Atencia, ed. José Jurado Morales, Madrid, Visor Libros, 2017, pp. 69-80.

10 Rafael León, Homenaje a Dioscórides, Madrid, Ínsula, 1976.

11 María Victoria Atencia, La señal. Poesía 1961-1989, ed. Rafael León, pról. Clara Janés, Málaga, Ayuntamiento, 1990, p. 52.

12 Joel R. Gômez, "Do campo científico para o campo literário: o diálogo entre metodologia de pesquisa e poesia na produçáo de Ernesto Guerra da Cal", en Avanços em literaturas e culturas africanas e em literatura e cultura galegas, eds. Petar Petrov, Pedro Quintino de Sousa, Roberto López-Iglésias Samartim y Elias J. Torres Feijó, Santiago de Compostela, Através Editora / Faro, Associação Internacional de Lusitanistas, 2012, pp. 307-325.

13 Antonio Gamoneda, Libro de los venenos, Madrid, Siruela, 1995. 


\section{Lo(s) SUSTANTIVO(s) EN LOS JARDINES}

Conviven dentro de este jardín verbal de Atencia lo humilde y lo sublime, con vistas a celebrar otro general, que más de una vez no es sino uno particular elevado a la categoría de jardín por antonomasia: desde el Retiro de Fray Alonso al parque de Málaga, que forman parte de su mundo más cercano, hasta jardines monumentales de otros lugares. En ocasiones incluso el mundo todo se presenta como jardín. La constitución de este corpus temático debe organizarse, por tanto, de modo análogo: como un recorrido por los nombres de las flores, arbustos y árboles, que son los que centran invariablemente la atención de nuestra poeta en cada texto.

Puede considerarse amplio su corpus acerca de este particular. En su obra son objeto de atención tanto los públicos como los privados. En otras ocasiones no se identifican, abriendo así una senda simbólica por la que transitará en más de una ocasión. Puede concretarse, no obstante, un atributo general: lo importante de los jardines son las especies que los habitan. No es la huella de la vida humana lo que prima en tales espacios, sino la verde vida que en ellos crece (y que engloba a la primera, dado que hablamos de jardines y no de selvas). Las arquitecturas, las huellas ornamentales, la piedra, pasan a segundo plano frente a la intimidad natural que los circunda y embellece.

El poema "Quintana" se abre de hecho con la teatralidad de los "plátanos orientales", acompañados de "bambúes" sobre un suelo cuajado de "hojarasca" ${ }^{14}$. Pero el afán de precisión léxica se materializa en un símil: "parecen las corolas grandes tazas de fuentes" (v. 4). Otro jardín público, "Retiro de Fray Alonso", es igualmente descrito de acuerdo con las especies que lo adornan: "magnolia”, "junquillos que crecen en un ánfora rota" o el "boj" se adueñan de los versos, mientras al fondo discurre el mundo ajado, acaso inerte ${ }^{15}$. Este distanciamiento (que no desinterés) del espacio arquitectónico se hace evidente en "Parador de San Francisco", donde la belleza de lo construido por el hombre aparece colonizada por

14 María Victoria Atencia, El fruto de mi voz, ed. Juan Antonio González Iglesias y bibliografía de Antonio Portela Lopa, Salamanca, Ediciones Universidad de Salamanca/Patrimonio Nacional, 2014, p. 53.

15 Ibidem, p. 275. 
el musgo, se erige en unidad temporal y física ${ }^{16}$. Al musgo que crece en la piedra se añaden unidades de serenidad representadas por el mirto y el laurel. Otra sede malagueña (la "Cuesta de la Reina") ejemplifica a las claras este protagonismo vegetal ${ }^{17}$. Alcornoques y lirios comparten con la voz poética la lucha contra el riguroso clima del invierno. Pero, aunque parecen pertenecer al mismo reino, se deben a climas distintos ("Estoy hecha a otra blanca cobertura salobre”, v. 4).

Todos los jardines monumentales mencionados en la poesía de Atencia reafirman su espíritu botánico. "Jardin du Luxembourg"18 contiene una significativa irrupción de lo divino, que adquiere una importancia simbólica a lo largo de toda su obra" ${ }^{19}$. "Natura invita" (v. 2), nos advierte, apuntando así una comunión entre vida y estética en dos versos no ajenos a lo sagrado: "hojas de gloria y frutos de un castaño / que da sentido a vida y a palabras" (vv. 8-9).

El boj es una de las plantas más citadas. En un "Jardín de Portugal” ${ }^{20}$ el humilde acto de la poda aparece de manera indirecta ("un reflejo verdea la tijera en el boj", v. 3), generando una imagen tan cinematográfica como ambigua. También el boj, plantado junto a un cenador que "se cuaja en glicinias" (v. 3) en el "Jardín de la Concepción"21, vale para construir un espacio simbólico donde la realidad se torna un déjà vu de percepción sublimada y mística: "poseída / —contra el cerco de bojpor el recuerdo / precisamente de este jardín y de su aroma” (vv. 6).

16 Atencia, La señal, p. 135.

17 Ibidem, p. 230.

18 María Victoria Atencia, La intrusa, Sevilla, Renacimiento, 1992, p. 23.

19 Sobre la presencia de este jardín parisino en la lírica española de las últimas décadas, remitimos a Guillermo Carnero, Fuente de Médicis, Madrid, Visor, 2006 (ahora en Guillermo Carnero, Jardín concluso, Madrid, Cátedra, 2020), que lo convierte en telón de fondo de la obra. Elide Pittarello, editora del volumen, refiere sobre su presencia en la obra del valenciano otro dato en sintonía léxica y conceptual con el poema de Atencia: "El canon renacentista se extiende [...] al jardín, donde el artificio del ser humano tenía que competir con la obra de la natura naturans" (Elide Pittarello, "Introducción", en Guillermo Carnero, Jardín concluso, pp. 154-155).

20 Atencia, La intrusa, p. 37.

21 Atencia, El fruto de mi voz, p. 308. Se trata del actual Jardín Botánico Histórico La Concepción, declarado en 1943 Bien de Interés Cultural por su interesante unión de arqueología y botánica. 
Dado el interés de Atencia por lo vegetal, el hecho de obviar el nombre de alguna planta se antoja significativo. Prefiere silenciar a sabiendas cuál ha encontrado en "Villa d'Este"22: "Por una tierna rama que muerdo y reconozco" (v. 1). Pertenece este a un grupo de poemas que tenderán a lo universal, motivo notable en los que se titulan genéricamente "Jardín". Es el caso del "Jardín" de Compás binario, cuya identificación solo se da en segundo plano mediante el subtítulo ("Rue Jean Rieux"). Aquí el léxico resulta menos riguroso, pero más preciso en clave poética, habida cuenta de que no identifica especies concretas ("árbol", "césped", "hojas"). Se compone a modo de grutesco de lo sustantivo. Este anonimato vegetal, sin embargo, es también el humano que se cita en la oscuridad del jardín.

Se hace necesaria, pues, una abstracción ${ }^{23}$. Apuntando a su lado espiritual, somete a la palabra "jardín" a este proceso, logrando así la máxima pureza significativa, la trascendencia del referente y del signo mismo: voz y recinto sagrado se confunden. En "Jardín" se lee: "por si yo misma fuera, acaso, tu jardín” (v. 10) ${ }^{24}$. Este mismo uso identificativo se repetirá en sus poemas más recientes, verbigracia "Tu imposible jardín": "Y cómo he de nombrarte, hallazgo mío, / para hacerme tu causa, tu imposible jardín" (vv. 1-2) ${ }^{25}$. A veces el jardín tiene la dimensión de un bosque ("El Bosque", De la llama en que arde), destacando "amentos de abedul, frondes de helecho" (v. 7) ${ }^{26}$.

Pero es en "El verde"27 donde Atencia conquista el grado de mayor abstracción. Se manifiesta la sustantivación del adjetivo más unido al universo de las plantas. Valiéndose del color igual que los impresionistas, se las arregla para personificar el verde, que "simplemente susurra, de

22 Ibidem, p. 286.

23 No hay que olvidar la larga tradición simbólica del jardín, a la que Atencia se acoge de un modo personal. Cirlot apunta que el jardín es "el ámbito en que la naturaleza aparece sometida, ordenada, seleccionada, cercada"; por ello "constituye un símbolo de la conciencia frente a la selva" (Juan Eduardo Cirlot, Diccionario de símbolos, Madrid, Siruela, 2007, p. 258). En esta sección son muchos los ejemplos en que parece dialogar y confundirse con el entorno. Llega a la plena identificación con los seres que lo pueblan en el poema "Jardín" del libro El hueco.

24 María Victoria Atencia, De pérdidas y adioses, Valencia, Pre-Textos, p. 9.

25 María Victoria Atencia, El umbral, Valencia, Pre-Textos, p. 24.

26 María Victoria Atencia, De la llama en que arde, Madrid, Visor, p. 21.

27 María Victoria Atencia, Las contemplaciones, Barcelona, Tusquets, 1997, p. 27. 
un modo clamoroso" (v. 3). La antítesis que contiene se define por su vitalismo. En otro poema muy relacionado, "Lo natural"28, parece responder al anterior cuando se pregunta: “¿De qué soy la carente si está ahí naturaleza? / Me renuevo en mis brotes, yo, la dócil” (v. 2). Los límites entre la voz de Natura y la de Atencia son aquí difusos.

En otras ocasiones, es el mundo entero el que se observa sub specie horti. Lo cual le permite recrearse en el detalle. Cualquier planta, flor o árbol que pueble la tierra es susceptible de deslizarse en un poema. Es numeroso el grupo no relacionados con jardines que tienen como protagonista las plantas o donde tan solo se las nombra. Destacaremos en este sentido "Árbol de Judea" 29 . Y tampoco se orille la multitud de veces en que florece la rosa (en el libro citado, por ejemplo, se incluye "La rosa”). La conspicua presencia de esta flor merecería, sin duda, un estudio aparte.

Un grupo especial lo forman aquellas plantas en las que la nomenclatura científica precede a la común, e incluso popular. Centrarán nuestra atención en los próximos epígrafes. Este paseo -forzosamente metonímico- por el jardín de María Victoria Atencia revelará una simetría perfecta entre nombre y planta en tres planos léxicos. El más preciso se corresponde, claro está, con la lengua científica. Cuando refiere la designación botánica, incide enseguida sobre la belleza del término y, por tanto, sobre la lengua en su grado superior; máxime cuando se trata de describir la naturaleza en sus seres más logrados. No menos exacto resulta el segundo plano, el de la lengua común: cada uno de nosotros evoca la flora de acuerdo con sus usos y condicionado por las variedades diatópicas ${ }^{30}$. El tercero es el lenguaje poético, mediante el cual extrema el rigor y la belleza de los anteriores, en una suerte de sincretismo. Solo en su genuino logos los tres planos pueden superponerse y trasladarse con naturalidad, en un ciclo comparable al de un ecosistema.

28 Ibidem, p. 35.

29 María Victoria Atencia, Trances de Nuestra Señora, Valladolid, Fundación Jorge Guillén, 1997, p. 34.

30 La cuestión de los diatopismos botánicos ha sido tratada por María de las Nieves Sánchez González de Herrero, "La adaptación del léxico botánico en las versiones castellanas de De Proprietatibus Rerum", Revista de Investigación Lingüística, XI, 1 (2008), pp. 287-305. 


\section{CHORISIA SPECIOSA}

El primer término que nos concierne es Chorisia speciosa, conocido comúnmente como "palo borracho". Dos particularidades distinguen a este árbol: 1) cuenta con una vistosa floración de intenso color rosado; 2) unas gruesas espinas llegan a cubrir el tronco y las ramas. Se trata de un árbol de hoja caduca originario de zonas tropicales, propicio, pues, para aclimatarse en la templada Málaga ${ }^{31}$. De ahí su pronto menudeo dentro de la poesía de Atencia. Lo mencionará dos veces, bajo distintas denominaciones. Por lo que al léxico se refiere, posee un triple interés, ya que es testigo del lenguaje común, del popular y del científico.

Chorisia speciosa es un basónimo (bautismo científico que se le debe a Augustin Saint-Hilaire). El poema, escrito no más tarde de 1979, por ańadidura, también vale la pena desde una perspectiva cronológica. Actualmente se lo clasifica como Ceiba speciosa, denominación propuesto por Pierfelice Ravenna en 1998.

Aparece por primera vez con la imagen popular en el breve poema "Palo borracho" 32 , seguida por su nomenclatura científica:

\section{Palo borRacho}

\section{Chorisia speciosa}

La temperamental constancia que da savia al árbol de los pájaros, emborracha de rosa a unas flores que anuncian esta luz del otoño.

La cercanía con este árbol se evidencia ya desde el título: elige el nombre común en Málaga, que parece derivar de la forma del tronco, más ancho en la base y con apariencia de botella. El interés por conservar el nombre común reside en que sobre el adjetivo vertebra el desarrollo del poema: la idea del nombre se amplía al imaginar la savia como un alcohol que

31 De hecho, pasa por ser uno de los árboles más representativos del Parque de Málaga. Gracias a Díez Garretas, sabemos que se trata de la especie que Atencia describe en el poema. Ver Blanca Díez Garretas, "Los palos borrachos del parque de Málaga", Boletín de La Academia Malagueña de Ciencias, 10 (2008), pp. 113-118.

32 Atencia, El fruto de mi voz, p. 154. 
sonrosa las flores mismas, como si de un beodo se tratase. Para apurar esta amplificatio alegórica, María Victoria Atencia añade luego el nombre botánico con una intención precisa: remontarse a un estadio original del lenguaje para sumar nuevas cualidades a la planta. Así, Chorisia deriva del apellido del ilustrador científico J. Louis Choris; pero es la voz speciosa la que entrańa mayor interés, en la medida en que su etimología y sonoridad debieron pesar en su consideración lírica. En la definición Raimundo de Miguel y Morante ${ }^{33}$, latinista y poeta del siglo XIX, reza:

Spěciōsus, a, um [de species].

Quint.Vistoso, bello, hermoso; Magnífico, ilustre; Especioso, aparente.

Es decir, a través del epíteto latino, Atencia ańade cualidades al palo borracho, y el nombre mismo puede revelarse como génesis del poema. Por otro lado, la definición de Miguel y Morante coincide con la registrada como primera acepción de la última entrega del Diccionario de Lengua Española de la RAE:

\section{especioso, sa}

Del lat. speciōsus.

1. adj. Hermoso, precioso, perfecto.

2. adj. Aparente, engañoso.

Recordemos que speciosa proviene de species, 'aspecto', 'semblanza'. Sería algo así como la forma, la figura, el aspecto, y viene a equivaler a la forma aristotélica, por un lado (lo que hace legible, visible e inteligible la materia), y a la belleza, por el otro, en tanto que dicha forma (speciosa) es bella, deslumbrante. Ahora bien, incluso la segunda acepción pudo estimular el poema, ya que se le ajusta como un guante al palo borracho; y más todavía al contraste entre su vistosa floración y la violencia de las espinas sobre su tronco y ramas. Es probablemente la definición de fondo para la escritura del segundo poema acerca de este árbol.

33 Raimundo de Miguel y Morante, Nuevo diccionario latino-espanol etimológico: con presencia de las obras más notables en este género publicadas en otros países... seguido de un tratado de sinónimos y de un vocabulario espanol-latino para uso de los jóvenes que frequentan nuestras escuelas, Madrid, A. Jubera, 1878, p. 872. 
Despojado ya ahora de su nombre científico, el palo borracho viene apelado mediante el americanismo "Samohú". La forma que recoge el DLE es samubú, y no menciona la variante aducida por Atencia:

Samuhú.

1. m. Arg. y Par. Palo borracho rosado.

Sí la documentan en cambio los corpus lingüísticos de la Academia. El CREA da noticia de dos testimonios de la variante "samohú", con marca diatópica argentina, mientras que para la forma que se halla en el DLE no trae ningún ejemplo. Son pocos los usos de "samuhú" registrados en el CORDE: solo cuatro concordancias, todas en Hijo de hombre, del paraguayo Roa Bastos ${ }^{34}$. Curiosamente, abunda más que "palo borracho", cuya búsqueda devuelve solo un resultado botánico entre un total de 1774. La preferencia por uno u otro, no obstante, no es decisiva porque conserva el consonantismo, cifra de la musicalidad exótica del sustantivo. Y son precisamente los nombres los que dominan el poema:

\section{SAMOHÚ}

Reconozco este árbol y su mudar continuo y comparto su voz desgarrada en las ramas hasta sentir astillas de madera mi sangre. Fugaces como un súbito deseo reprimido o el hueco que en el aire deja el paso de un pájaro, soy ya vuestra querencia, palabras floreales de una amarga balada para emprender el sueño ${ }^{35}$.

El uso de sendos nombres comunes, que hacen las veces de títulos, indica el deseo de sublimar la terminología botánica, amén del tránsito natural de la poeta por todos los senderos de la lengua.

34 Real Academia Española. Banco de datos (CORDE) [en línea]. Corpus diacrónico del español. <http://www.rae.es> (consultado el 25/02/2021). Augusto Roa Bastos, Hijo de hombre, Madrid, Alfaguara, 1977.

35 Atencia, El fruto de mi voz, p.183. 


\section{ARUM DRACUNCULUS}

A guisa de seudo-díptico con "Palo borracho", el poema "Dracúnculo"36 recicla el lenguaje botánico de manera bien distinta. Se diría que la elección de términos en este caso es una opción de la escritora, y la que da nombre al poema la definitiva. Como en el caso del primer texto del anterior bloque, Atencia refiere el nombre científico de Arum dracunculus, propuesto por Carlos Linneo en 1753. Pero de nuevo elige un basónimo latino. En la actualidad, se utiliza el más extendido Dracunculus vulgaris, cuyo bautismo corrió a cargo de Heinrich W. Schott en 1832. Luego volveremos sobre esta preferencia. No obstante, "dracúnculo" tampoco es hoy el nombre común más extendido, sino el de "dragontea". Natural de Europa, suele hermosear jardines y zonas húmedas. Se singulariza por su espátea abierta, con forma cónica, y un interior color púrpura desde el cual se yergue un espádice carmesí. Se trata de una planta tóxica, aunque se le atribuyen propiedades cosméticas y dermatológicas para el tratamiento de las pecas y las manchas faciales ${ }^{37}$.

Detengámonos en la etimología del nombre botánico elegido por Atencia. El primer término, “arum”, proviene del griego äpov, o sea, el aro egipcio, especie de la misma familia que la dragontea ${ }^{38}$. Por su parte, dracunculus, diminutivo de draco ('dragón'), posee un par de acepciones útiles para nuestros fines: por un lado, lo define como "serpiente pequeña”, y por otro, según anotara Plinio, como "dragoncillo, estragón, yerba" 39 . Aunque recibe muchos nombres populares, el DLE apenas recoge cuatro: "dragontea”, "culebrilla”, "serpentaria” y "taragontía”. La búsqueda de los tres últimos remite directamente al primero ${ }^{40}$.

36 Ibidem, p. 155.

37 Dioscórides señala que "los que se frotan las manos con ellas o cogen la raíz, no sufren mordeduras de víbora" (Dioscórides interactivo [en línea] < https://dioscorides.usal.es/p2.php?numero=366> (consultado el 09/02/2021), aspecto que quizá pueda vincularse con uno de sus nombres populares. Empero, es una planta tóxica; un detalle que, según se verá, incide sobre el poema de Atencia.

38 Ibidem.

39 Miguel y Morante, op. cit., p. 309.

40 Así la define la última edición: "Del lat. dracontēa, y este del gr. $\delta p \alpha \kappa o v \tau \varepsilon i ́ \alpha$ drakonteía. 1. f. Planta herbácea vivaz, de la familia de las aráceas, de rizoma feculento y grueso, del cual arrancan hojas grandes divididas en cinco lóbulos lanceolados, con 
Como hemos indicado, las elecciones léxicas suponen cuestiones de primer orden en su poética. En primer lugar, la predilección por Arum dracunculus, y no por Dracunculus vulgaris, responde a un argumento de prestigio. Atencia prefiere el basónimo para evitar el adjetivo vulgaris, menos enigmático que arum. No descartamos la hipótesis de que también homenajee a Linneo como garante de la lengua científico. No en balde, la poesía codicia nombrar el mundo ex nibilo. Así se desprende del asombro y la inquietud que despierta la contemplación de esta planta:

\section{DRACÚNCULO}

\section{Arum dracunculus}

De no sé qué país, perturbadoramente alzada, flor color del olvido o la escoria ${ }^{41}$, la belleza es bastante razón para tu muerte; tu podredumbre, un nuevo testimonio de vida.

Como sucedía con el palo borracho, la dragontea es objeto de un segundo texto. Resucita dentro de la serie "Paseo de la farola"

\section{"FLOR"}

La dragontea aguarda en el barro cocido de su maceta al día. Y allá abajo, en las aguas,

pecíolos anchos que abrazan el escapo, simulando un tallo de 60 a $80 \mathrm{~cm}$ de altura, manchado de negro y verde como la piel de una culebra, espata grande, verdosa por fuera y purpúrea negruzca por dentro, y espádice largo y desnudo en su extremo, que se cultiva como adorno en los jardines, a pesar de su mal olor durante la floración, y es espontánea en varios puntos de España”.

41 El sintagma "flor color de olvido" encierra un claro guińo a Cernuda: "Furia color de amor / amor color de olvido" ("La canción del oeste", en Luis Cernuda, Un río, un amor, 1929, recogido en La realidad y el deseo, Madrid, Cruz y Raya, Ediciones del Árbol, 1935). Lorca usa esos dos versos como lema de la sección "Poemas de la soledad en Columbia University" de su Poeta en Nueva York (1929-1930).

42 Atencia, La señal, p. 180. 


\section{"Fleur" -¿qué flor?-, un dragón \\ hay que ostenta ese nombre \\ sobre su hierro negro \\ con grandes letras rojas. \\ La France, la France: un aire \\ de fleur despide el puerto.}

Las comillas del título, modificadores del sustantivo, lo extienden a dos planos simultáneos de la realidad. Por un lado, a la realidad inmediata, cercada en el barro cocido donde reposa la dragontea; por el otro, al plano metafórico-mitológico, atraído por el nombre de la planta y por el del barco, cuyo nombre, entrecomillado, también podría aplicarse a la extraña forma de la dragontea. Atencia dibuja así dos paisajes con un solo nombre.

\section{ANASTATICA HIEROCHUNTICA}

El siguiente nombre nos guía por un itinerario a través del desierto y el tiempo. Se trata de la Anastatica hierochuntica, más conocida por "rosa de Jericó”. Se trata de una fascinante planta capaz de resistir las duras sequías de Oriente Medio y el Sáhara gracias a la trehalosa, un tipo de azúcar. Se pliega sobre sí misma hasta hacerse un ovillo, lo cual le permite ser arrastrada por el viento con facilidad. Esta defensa la ayuda a sobrevivir durante años en estado letárgico, hasta que de nuevo entra en contacto con el agua. Fruto de la rehidratación, comienza entonces a abrirse y a recobrar su verdor. Dicho proceso puede repetirse varias veces, por lo que se la tiene por un símbolo de renacimiento. Su nombre científico, invariable desde los tiempos de Linneo, se basa en esa cualidad. La voz anastatica, proveniente del griego $\dot{\alpha} \nu \alpha \dot{\sigma} \tau \tilde{\alpha} \sigma \iota \varsigma$, significa 'resurrección'43. Hierochuntica es el adjetivo derivado de Hiericho (Jericó), aunque parece que la relación con la ciudad Palestina es más legendaria que botánica.

El DLE hace referencia en su definición de "rosa de Jericó" a su principal característica:

43 Diccionario Griego-Español. Instituto de Lenguas y Culturas del Mediterráneo y Oriente Próximo Centro de Ciencias Humanas y Sociales, CSIC. <http://dge.cchs. csic.es/xdge/ $\alpha \dot{\alpha} \alpha \tau \tilde{\alpha} \sigma \varsigma \varsigma>$ (consultado el 19/02/2021). 
1. f. Planta herbácea anual, de la familia de las crucíferas, con tallo delgado de hasta $20 \mathrm{~cm}$ de altura y muy ramoso, hojas pecioladas, estrechas y blanquecinas, y flores pequeñas y blancas, en espigas terminales, que vive en los desiertos de Siria, y al secarse las ramas y hojas se contraen formando una pelota apretada, que se deshace y extiende cuando se pone en agua, y vuelve a cerrarse si se saca de ella.

Retengamos las etapas de tan particular proceso de rehidratación, porque sobre ellas se levanta el poema que le consagró Atencia:

\section{RosA DE JERICó \\ Anastatica hierochuntica}

Tantos años, y más, dejada en el armario, con luz escasa y sed y savia detenida, un vaso de cristal de Suecia interrumpe.

Tersos rasgos se yerguen

que asaltan con sus brincos de nuevo los gorriones, ajenos a esta tregua entre la sed y el agua ${ }^{44}$.

El sintagma de apertura ("tantos años") se intensifica mediante "y más", fórmula imprecisa que sugiere la tozuda supervivencia de la planta, al tiempo que brinda pistas de la modulación del discurso poético. La operación léxica deviene rica en lecturas. En el v. 2 el vocabulario botánico se ve sometido a un proceso de poetización, dado que traduce la trehalosa como "savia". Pero, como ya hemos tenido oportunidad de comprobar, el hecho de que prefiera un término menos preciso en lo botánico -si bien de mayor vuelo lírico- no implica desconocimiento, según se deduce del epíteto "detenida". La única voz repetida es "sed", que simboliza la rehidratación. El poema también es susceptible de contemplarse bajo la luz de la alegoría, si se identifica la rosa de Jericó con los ciclos vitales.

44 Atencia, El fruto de mi voz, p. 184. 


\title{
6. VENERI (GRATISSIMA) MYRTUS
}

Uno de los hitos de este lenguaje científico se cifra en "Le temps" ${ }^{45}$, donde la nomenclatura botánica puede calificarse de ida y vuelta:

\section{Le Temps}

\section{Veneri gratissima myrtus}

\author{
Asaltando los mirtos \\ en celestiales torres \\ para el amor alzadas, \\ le temps, le temps s'en va.
}

El mirto se alza aquí como testigo del tiempo, construyendo una fortificación inseparable de aquellas a las que escolta. La clave del engarce entre arquitectura y vegetación estriba en el verso "para el amor alzadas". A tenor de lo dicho en páginas anteriores, podría dar la impresión de que el título responde al nombre botánico del arrayán. Y sin embargo, Veneri gratissima myrtus es literatura en estado puro ${ }^{46}$. El sintagma proviene de la mitología, ya que se asociaba con la diosa Venus (apodada Myrtea) ${ }^{47}$. Al contrario que en ejemplos previos, en este poema se nombra poéticamente lo que debería ser científico. Atencia le da un nuevo giro a las bases de la taxonomía, propiciado, sin duda, por textos anteriores. El juego léxico se refuerza ahora por dos veces, si se tiene en cuenta uno de los sinónimos por que se conoce la planta. Si bien el ámbito botánico ha primado Myrtus communis, la misma especie fue bautizada como Myrtus veneris por Bubani en el siglo xIx, que acudió a las mismas fuentes literarias que la malagueña. Se antoja un punto de partida plausible que hunde sus raíces en la poesía romana. Acaso haya que buscar en Virgilio el origen de tan soberbia operación lingüística:

45 Ibidem, p. 164.

46 Sobre el mirto en las letras latinas remitimos a Esteban Bérchez Castaño, "Mirto y ajenjo en la poesía romana", Minerva Revista de Filología Clásica, 23 (2010), pp. 127-142, quien advierte que acostumbra a asociarse al locus amoenus, motivo de fondo en el poema de Atencia.

47 Louis de Jaucourt, Encyclopédie ou Dictionnaire raisonné des sciences, des arts et des métiers, París, André Le Breton, 1751. La entrada reza: "MYRTEA, (Mythol.) Apodo de Venus, por el mirto que se le dedicó Formosae Veneri gratissima myrtus". 
C. Populus Alcidae gratissima, uitis Iaccho, formosae myrtus Veneri, sua laurea Phoebo;

Phyllis amat corylos: illas dum Phyllis amabit, nec myrtus uincet corylos, nec laurea Phoebi ${ }^{48}$.

La relación intertextual del poema de Atencia con estos versos del mantuano se enmarca a su vez en un par de géneros. El pasaje está tomado de las Bucólicas, y no de las Geórgicas, que sería la obra más cercana al campo botánico. Se asocian también dos lenguajes distintos, el científico y el poético, de los cuales el segundo acaba sustituyendo al primero. Fray Luis de León tradujo este pasaje evidenciando los lazos entre el mirto y Venus, unión inseparable cuyo origen se pierde en el tiempo:

\section{CORIDÓN}

El álamo de Alcides es querido, de Baco la vid sola es estimada, el mirto de la Venus siempre ha sido, y en el laurel por Febo es Dafni amada;

el córilo es de Filis escogido, del córilo la Filis pues se agrada; al córilo conozcan por rey solo el mirto y el laurel del rojo Apolo (vv. 113-120) ${ }^{49}$.

48 De la edición de Aurelio Espinosa Pólit, Virgilio en verso castellano: Bucólicas, Geórgicas, Eneida, Ciudad de México, Clásicos Universales Jus, 1961, p. 44. Plinio el Viejo, Historia Natural (XII-XVI), trad. y notas de Francisco Manzanero Cano, Ignacio García Rivas, María Luisa Arribas Hernáez, Ana María Moure Casas, José Luis Sancho Bermejo, Madrid, Gredos, 2010, p. 8, enumera estas correlaciones en su Historia natural, pero apunta que son las divinidades las protectoras de tales plantas: "hay especies arbóreas que gozan de permanente de protección por estar consagradas a determinadas divinidades, como el roble a Júpiter, [...] el mirto a Venus y el álamo a Hércules.

49 Fray Luis de León, Poesías, ed. Javier San José Lera, Biblioteca Virtual Miguel de Cervantes [en línea]:<http://www.cervantesvirtual.com/obra-visor/ poesias--3/html/01e9471c-82b2-11df-acc7-002185ce6064.html> (consultado el 23/01/2021). 
Concedemos que son muchos los testimonios que repiten tout court la cita que nos atañe. Uno de los más llamativos se localiza en la primera edición de la Encyclopedie ("Formosae Veneri gratissima myrtus"). Pero, más allá de la asociación pagana, hay una cuestión nada tangencial si se considera la obra de la malagueña. El mirto es también un símbolo mariológico ${ }^{50}$. Si bien el subtítulo parece apuntar a un sustrato pagano, el adjetivo "celestiales" abre las posibilidades de significación hacia lo mariológico.

Igual que ocurrió con el dracúnculo, en la poesía de Atencia asoma de vez en cuando el nombre popular de esta planta. La más significativa es la mención del arrayán en su obertura a la más monumental de las puertas de la Alhambra: la de la Justicia. Mientras camina hacia ella, va enumerando las plantas que halagan los sentidos:

Puerta de la Justicia

Llevarme el arrayán perdida hasta tus patios, olvidarme el ciprés, agredirme un aroma de violeta, negarme su artesonado el cedro, romper la luz el agua quebrada de tus fuentes, rasgarme pecho abajo un pájaro que cruza, amargarme tu aliento de granado en la boca sin siquiera vivirme, sin tan volver siquiera ${ }^{51}$.

El arrayán, el ciprés, la violeta, el cedro y el granado conforman una selva donde cada uno de los sentidos encuentra su cumplimiento. Es un ejemplo también de poema altamente simbólico. En ese plano la orografía del terreno que acoge la Alhambra es un dato de primer orden. Como es sabido, para acceder a la Alhambra se debe subir alguna de las cuestas que la circundan. Es decir, el camino en ascensión, cuajado de flores y árboles, hace que este poema se revele, leído en clave alegórica, como descripción de un súbito paraíso hedonista.

50 Recordemos, para estos propósitos, un verso de la letanía compuesta por el abad Adam de Saint-Victor: "Mirto de templanza, rosa de paciencia, nardo fragante". Apud Augusto Nicolas, La Virgen María viviendo en la Iglesia. Nuevos estudios filosóficos sobre el cristianismo, trad. José Vicente y Caravantes, Madrid, Manuel Minuesa, 1861, p. 234.

51 Atencia, La señal, p. 324. 


\section{Datura Suaveolens}

La última planta se conoce popularmente como floripondio, aunque el DLE también recoge campana y borrachero. No obstante, aunque el diccionario no refiera su nomenclatura, los tres se corresponden con varias especies de brugmansias, como la candida y la misma suaveolens. Sin embargo, hay cierto debate sobre si cabe hablar de dos géneros: brugmansia y datura ${ }^{52}$. Así las cosas, el concepto botánico elegido por Atencia es el segundo, lo cual vuelve a tener secuelas poéticas. El primero de los nombres representa un tributo a Sebald Justinus Brugmans y el segundo deriva del participio de futuro del verbo latino dare ("la que va a dar" $)^{53}$. El epíteto suaveolens viene definido por Raimundo de Miguel como "lo que tiene olor suave, grato" 54 . De modo que la inclinación por este nombre se deriva aquí de la semántica, pero también de la fonética:

\section{Datura SUAVEOLENS}

Prosigue la datura en su aroma caudal, ya en la senda del tacto.

Conozco su perfume:

necesité mi vida

para poder coserlo

con agujas de hielo, flor carnal, a mi carne (vv. 1-6)

La evocación del nombre alcanza a varios órdenes correlativos. En el lingüístico, es la fonética la que se impone como argumento para elegirlo por encima del antedicho. Todas y cada una de las consonantes de Datura suaveolens se articulan en una zona reducida de la boca (dientes, alveolos y labios), lo que invita a pensar en una evocación fonosimbólica del persistente aroma que desprende su flor. La secuencia consonántica se une así al sentido del gusto, como si se retuviera su nombre gracias a su fragancia, como sucede con los perfumes oleosos. El reino de los sentidos

52 Se documentan otros nombres usuales como trompetero amarillo, trompeta del juicio amarilla o floripondio blanco, aunque la Academia no los recoge.

53 Miguel y Morante, op. cit., p. 256.

54 Ibidem, p. 887.

55 Atencia, El fruto de mi voz, p. 162. 
es, no por casualidad, el otro protagonista de este poema, como se publica en el segundo verso. Y tampoco se orille la toxicidad de esta planta. No en vano, la lengua popular la bautizó como borrachero, y se han descrito episodios de midriasis a causa de la sola visión de esta planta. El contacto con ella puede producir alucinaciones, ya que entre sus compuestos se cuenta la escopolamina, uno de los ingredientes de la burundanga.

\section{Conclusiones}

La obra completa de María Victoria Atencia encierra en su interior un jardín personal, al que canta, palabra por palabra, en los nombres de las plantas. De acuerdo con la función poética del lenguaje, lo conforman otros tres jardines de Málaga que deben de haber influido en su escritura y en el formato verbal que utiliza para describirlos. Dos de ellos son jardines botánicos y dan pie a un género discursivo definido por el hecho de contener lenguaje.

En primer lugar, el Jardín Botánico, todavía visible en los años treinta y cuarenta, llegó a ser uno de los mejores de Espańa. Es el botánico por antonomasia en el imaginario de la andaluza ${ }^{56}$. El segundo atiende por Jardín Botánico Histórico La Concepción, al que le dedica "Jardín de la Concepción”, prueba de su magnífica conservación a lo largo de las décadas. Lo interesante desde el punto de vista botánico-poético se desprende de su organización del catálogo de especies. El tercero es el Parque de Málaga, que forma parte de su mundo más cercano. También se trata del parque por antonomasia. En él crecen algunas de las plantas nombradas en estas páginas. Por ejemplo, el palo borracho: en concreto en la zona hoy conocida como Jardines de Alfonso Canales. Hablamos, por tanto, de un árbol dos veces poético.

56 Blanca Lasso De la Vega y Alfredo Asensi Martie, "Un verdadero jardín botánico del siglo XXI en la Málaga del XIX”, Isla de Arriarán: Revista Cultural y Científica, 34 (2009), pp. 159-184, recogen un testimonio de Temboury sobre el estado en que se hallaba el Botánico en 1947: "El jardín, que, como tantas otras cosas del edificio, muere en el más lamentable abandono, apenas deja ver su trazo, ya que sólo se percibe un hermoso cenador de hierro con grandes corta-fuertes forjados en cuerno de carnero. En medio del cenador existe una fuente de pitón estrellado y gallonada taza que rodean 7 bancos de piedra, cerrajería y cerámica” (p. 181). 
Hemos estudiado en profundidad el breve corpus de plantas que llevan como título o subtítulo un nombre botánico. No por breve deja de ser exhaustivo: son todas las que ponen en práctica dicho proceder: nombre botánico de dos términos (género y especie), seguido de la descripción (el poema). Nuestro análisis de la nomenclatura evidencia que el jardín verbal de Atencia se diseña como un jardín botánico. Es una colección de plantas y flores reunidas. Un detalle que inevitablemente remite al valor etimológico de "antología" ('ramillete selecto'). Sin embargo, Atencia no corta flores para hacer un bouquet. Su colección equivale a un jardín botánico verbal con plantas de diversas latitudes, seleccionadas en razón de su belleza y de su gusto. Igual que en un jardín botánico, el lector contempla las flores (los nombres de las flores) y se topa con las oportunas cartelas, casi fichas científicas convertidas en epigramas. El discurso de las descripciones lexicográficas se transforma en écfrasis tan connotativas como sensuales. Cada nombre se renueva, a veces recuperando su etimología o la raíz mitológica. También, si llevasen una ilustración (dibujo o fotografía), se asemejarían a las tres partes de un emblema: lema, pictura y epigrama.

$\mathrm{Su}$ agudeza aplica la función poética a los nombres mismos de las plantas, tanto a los comunes como los que provienen de la botánica. En ambos casos los ajusta a una realidad (re)nombrada y por ello recreada: el jardín con su Paraíso.

Nuestro método lexicológico nos faculta para concluir que todos los nombres de flores, árboles y plantas pudiesen ser vistos en potencia acompañados de sus respectivos correlatos científicos, convirtiéndose en poéticos e implicando a los cinco sentidos en la lectura/visión, no exenta de ética. Detengámonos siquiera en el narciso de "Entre los que se fueron" ${ }^{57}$ :

Narcisos dejaré más allá de esta hora

y que toquen sus pétalos nombres entrelazados (vv. 7-8).

El "narciso común" o "de los jardines" fue bautizado como Narcissus poeticus en el Species Plantarum (1753) de Linneo. Este nombre, que suele traducirse por "narciso de los poetas" funciona como metonimia del

57 Atencia, La señal, p. 8. 
modo en que Atencia percibe las plantas para construir un jardín botánico cuyo itinerario se traza a medida que lo describe. La poesía completa de la malagueña merecería aquella etiqueta que Linneo le puso al narciso y el análisis lexicológico que Callebat reservó para la rosa.

Naturaleza, ciencia y lenguaje nos recuerdan que el mundo es un jardín. Por eso cobra tanto simbolismo el poema "Jardinero mayor" 58 , donde este oficio se ilustra también en clave figurada, a resultas del continuo abrazo con la tierra: "me encontraron un día / a ella abrazado como quien engendrara un hijo" (vv. 11-12). Es la expresión más viva de que lo poético del mundo reside en su calidad de codex vivus naturae ${ }^{59}$; de aquí que toda la obra de Atencia venga signada por la sinonimia entre libro y jardín. Tal vez pueda resumirse esta invitación al logos desde el fondo de lo vegetal con estos versos de Eliot:

Y otros ecos

habitan el jardín. ¿Vamos tras ellos? ${ }^{30}$

58 Ibidem, p. 27.

59 El término proviene de la filosofía del Seiscientos italiano, concretamente de Raymundo Desabunde, como se refiere en Santiago Beruete, Jardinosofia: una historia filosófica de los jardines, Madrid, Turner, 2016, p. 111. Beruete distingue dos líneas renacentistas en la observación de los jardines, con repercusiones lingüísticas. Una se define de corte más empírico, y la otra como hermética. Consideramos que Atencia las aúna en su poesía, donde el lenguaje científico se reviste de cierto hermetismo semántico.

60 T. S. Eliot, "Burnt Norton", en Cuatro Cuartetos, trad. José Emilio Pacheco, Ciudad de México, Fondo de Cultura Económica, 1989, pp. 9-10. 